\title{
Stochastic characterization of the spectrum sharing game in ad-hoc networks
}

\author{
Ilaria Malanchini ${ }^{\mathrm{a}, *}$, Steven Weber $^{\mathrm{b}}$, Matteo Cesana ${ }^{\mathrm{c}}$ \\ a Bell Labs, Alcatel-Lucent, Germany \\ ${ }^{\mathrm{b}}$ Electrical and Computer Engineering Department, Drexel University, Philadelphia, United States \\ ${ }^{\mathrm{c}}$ Dipartimento di Elettronica, Informazione e Bioingegneria, Politecnico di Milano, Italy
}

\section{A R T I C L E I N F O}

\section{Article history:}

Received 10 August 2014

Received in revised form 5 December 2014

Accepted 19 January 2015

Available online 11 February 2015

\section{Keywords:}

Wireless access networks

Spectrum sharing

Non-cooperative game theory

Stochastic geometry

\begin{abstract}
A B S T R A C T
This work focuses on infrastructure-less ad hoc wireless networks where multiple transmitter/receiver pairs share the same radio resources (spectrum); transmitters have to choose how to split a total power budget across orthogonal spectrum bands with the goal to maximize their sum rate under cumulative interference from concurrent transmissions. We start off by introducing and characterizing the non-cooperative game among transmitter/receiver pairs when the network topology is deterministically given. The corresponding Nash equilibria are derived, highlighting their dependency on the topological parameters (distances between wireless nodes, propagation model, and background noise power). The analysis is then extended to the case of random network topologies drawn from a given spatial stochastic process. Tools of stochastic geometry are leveraged to derive a statistical characterization of the equilibria of the spectrum sharing game. Finally, a distributed algorithm is proposed to let the players of the spectrum sharing game converge to equilibria conditions. Numerical simulations show that the proposed algorithm drives the users to stable points that are close to the equilibria of the game requiring limited information exchange among nodes.
\end{abstract}

(c) 2015 Elsevier B.V. All rights reserved.

\section{Introduction}

Resource sharing problems naturally arise in case of wireless networks where the transmission medium is inherently broadcast and transmission resources, e.g., frequency channels, transmission power levels, temporal slots, have to be orchestrated among multiple concurrent transmissions. The general goal is the design of sharing algorithms/protocol to maximize the "usage efficiency" of the shared resources.

\footnotetext{
This work extends our preliminary study presented in [1,2].

* Corresponding author. Tel.: +49 711821 30259; fax: +49 711821 32185.

E-mail address: Ilaria.Malanchini@alcatel-lucent.com (I. Malanchini).
}

In this work, we focus on infrastructure-less ad hoc wireless networks where multiple transmitter/receiver pairs share the same radio resources (spectrum). Each pair is allowed to allocate an available transmission power budget across multiple radio resources for transmission (reception). Such scenario well represents those practical cases where either multiple Network Interface Cards (NICs) are available at each transmitter/receiver [3] or where the transmission technology allows to use different power levels on different subcarriers, like the IEEE 802.11a OFDMA-based technology [4]. In any case, the "quality" perceived by a transmitter/receiver pair is influenced by the behavior (resource allocation) of the other users. The reference scenario is inherently distributed as there is no central entity to coordinate the resource allocation network-wide, but rather each transmitter/receiver pair 
adopts local resource allocation policies depending of its local view and knowledge of the network. In this view, our main goals are to tell whether (and when) there exist equilibrium policies which are stable; whether these equilibria are unique; and whether they can be reached by distributed algorithms. To this extent, we model the interaction among users as a non-cooperative game.

In particular, we consider a Gaussian Interference Game (GIG) [5], where multiple transmitters have to decide how to split a total power budget across orthogonal spectrum bands with equal bandwidth; the users play to maximize their sum rate over all the used bands which depends on the interference level produced by the other users and some background Gaussian noise. Since the wireless network performance is strongly influenced by the spatial distribution of the communicating/interfering nodes, a natural objective is to analyze the dependency of the equilibria distribution on the node positions. To this extent, we start off by introducing and characterizing the non-cooperative game where the network topology is deterministically given, that is, the relative positions of transmitters and receivers are known. We take here a constructive approach by analyzing at first a simple but insightful network topology with two transmitter/receiver pairs. In this scenario, we derive the quality/number of the Nash equilibria, highlighting their dependency on the topological parameters (distances between wireless nodes, propagation model, and background noise power). We show that the game solution features a bi-modal behavior in which if the two pairs are "far enough" the equilibrium is unique and coincide with the optimal centralized-based allocation, whereas if the two pairs "get closer" the game admits multiple equilibria that in general do not coincide with the optimum.

The paper then moves to the characterization of the non-cooperative spectrum sharing game for random network topologies drawn from a given spatial stochastic process. We leverage tools of stochastic geometry to derive a statistical characterization of the equilibria of the spectrum sharing game. Again, we start off by providing the analysis of the equilibria in the two transmitter/receiver pairs scenario; we further show how the analysis of the 2-player game can be leveraged to characterize the $\mathrm{N}$-player game. Namely, the analysis of large networks game can be simplified, by wisely decomposing the $\mathrm{N}$ player game into independent equivalent sub-games of two players. A heuristic algorithm is proposed to implement such decomposition, thus allowing to get the equilibria for the $N$-player game. Finally, a distributed algorithm is introduced to let the $N$ players converge to equilibria conditions. Numerical simulations show that the proposed algorithm drives the users to stable points that are close to the equilibria of the game further requiring only minimal information exchange.

In short, the main contributions of the present work can be summarized as follows:

1. Analysis of the 2-player spectrum sharing game.

(a) Characterization for deterministic topologies of the Nash equilibria, their stability and quality with respect to the optimal solution. (b) Analysis of the spectrum sharing game in stochastic topologies.

2. Analysis of the $N$-player spectrum sharing game.

(a) Proof of existence of at least one Nash equilibrium in pure strategy.

(b) Analysis through directed influence graph approach and coupling probability.

3. Distributed algorithm to drive the selfish user to play equilibria strategies in spectrum sharing.

The paper is organized as follows: in Section 2 we review prior work on game theoretic approaches to spectrum/resource sharing in wireless networks; Section 3 sets the reference scenario; Section 4 provides a detailed analysis of the 2-player game; Section 5 extends the analysis to the $N$-player game; Section 6 provides distributed algorithm to reach equilibrium and compare the result with the game solutions; and Section 7 concludes the paper.

\section{Related work}

The relevant literature includes work on game theoretic tools and stochastic geometry applied to network scenarios where network nodes have to optimally allocate transmitted power across multiple (semi-) orthogonal bands with target of maximizing the achievable throughput. Such problem is indeed relevant in different networking scenarios including ad hoc networks, cognitive radio networks and wireless access networks. In the following, we give a succinct overview of the main findings and approaches related to these three application scenarios. In [6], the authors consider a power control problem with SINR as objective function, in both selfish and cooperative scenario. In the selfish case, the users play to maximize their achievable average SINR over all the resources they use to transmit; existence and "quality" of the Nash equilibria are studied under different cases for mutual interference between the users over the available resources. In the cooperative scenario, the users play to maximize the sum average SINR.

Wang and Krunz consider in [7] a non cooperative scenario where users compete in the power allocation to maximize their performances. A pricing mechanism to steer the non-cooperative spectrum sharing process towards optimal equilibria is proposed.

Power games based on the water-filling algorithm are proposed in $[8,9]$. The authors consider a scenario composed by two contending communicating systems and study the existence and uniqueness of the Nash equilibrium. The water-filling algorithm is used also in $[10,11]$. A unified view of main results presented in the literature is proposed in [12]. This work shows how the different approaches proposed in the literature can be unified following a unique interpretation of the water-filling solution. Furthermore, a unified set of sufficient conditions that guarantee the uniqueness of the equilibrium is derived. Baidas and McKenzie [13] leverage auction mechanisms to properly set the transmission power levels in multisource, multi-relay wireless networks in a cooperative environment. 
Power control games in the context of cognitive radio networks are considered in [14-16]. Existence and properties of the Nash equilibria are investigated assuming the interference temperature model.

In [5], a Gaussian Interference Game (GIG) is proposed, in which a player can allocate a fixed amount of power arbitrarily across a continuous bandwidth with the aim to maximize its sum rate under Gaussian interference. The authors further propose self-enforcing rules that allow users to reach an efficient and fair solution. Different from our case, the available bandwidth is considered as continuous, whereas we consider the case in which discrete frequency channel can be used.

Spatial reuse is explicitly accounted for in the spectrum sharing process in the so called spatial congestion games, which model the competition among secondary users of a cognitive radio network in accessing spectrum opportunities [17,18] and references therein. In [17], the congestion game is played on undirected interference graph, assuming symmetric wireless links, whereas [18] extends the model to the case of directed interference graphs. Existence of Nash equilibria and distributed convergence algorithms are proposed in both works.

Two system pairs sharing two frequency bands are considered in [19]. For three different relaying strategies, the existence and the uniqueness of the equilibrium are analyzed. The general scenario with multiple transmission pairs is addressed in [20], where the power allocation game is modeled as a potential game that is shown to possess a unique equilibrium. When the channels are assumed to fluctuate stochastically over time, stochastic approximation theory is used to show convergence to equilibrium. Different from our case, the aforementioned two papers assumes that the two transmitters can exploit the presence of a common relaying node.

The competition for accessing the available radio resources may as well happen among wireless networks as a whole, rather than among single wireless links, as considered in the aforementioned papers [21-23]. In [22], the authors study the competition between two wireless access networks operating according the IEEE 802.22 standard. The problem is cast as a non-cooperative power game in which each player aims at maximizing its coverage by increasing its transmission power while taking into account the interaction with the competitor which happens through reciprocal interference. In [23], a Stackelberg formulation of the spectrum sharing game is proposed where the two operators are prioritized in playing their strategies.

To wrap up, in this work we generally advance the literature on power allocation in wireless networks by coupling tools of game theory and stochastic geometry [24]. To the best of our knowledge, ours is one of the first attempts to propose a stochastic analysis of noncooperative games in the context of wireless networks. A notable exception is represented by the work in [25], where the authors propose a stochastic game that models the interactions among users competing for the available spectrum opportunities. However, the game used in [25] is different then ours as it is based on an auction in which users make bids for the required resources.

\section{Scenario}

We use standard probabilistic notation: sans-serif letters (e.g., $\mathrm{x}$ ) denote random variables (RV) and italic characters denote their corresponding value (e.g., $x$ ). The cumulative distribution function (cdf), its complement (ccdf), and probability density function (pdf) are denoted $F_{\mathrm{x}}(x), \bar{F}_{\mathrm{x}}(x)=1-F_{\mathrm{x}}(x), f_{\mathrm{x}}(x)$, respectively, with the natural notational extensions for conditional and joint distributions.

\subsection{Game model}

We consider a Binomial Point Process (BPP) $\Pi_{N}=\left\{\mathrm{u}_{1}, \ldots, \mathrm{u}_{N}\right\}$ where $N$ transmitters are positioned independently and uniformly at random in a convex set $\mathcal{A} \subset \mathbb{R}^{2}$ with $N /|\mathcal{A}|=\lambda$; we refer to $\lambda$ as the density. The $N$ receivers $\left\{\mathrm{v}_{1}, \ldots, \mathrm{v}_{N}\right\}$ are each positioned independently and uniformly at random on the circle of radius $d_{i}$ centered at the corresponding transmitter. We choose the BPP model since it is the most natural model when no concrete information on the user distribution is available. Furthermore, it has the advantages of being simple, tractable, and consistent with prevailing use in the literature. Fig. 1 reports an example of the considered topology.

We consider the spectrum sharing game, where the $N$ transmitters (the players) have to decide how to split the total power budget over the available spectrum (the actions). Namely, we assume that the spectrum is divided into two bands, which we label the left band and right band. The strategy space of the generic transmitter $i$ is $P_{i} \in[0,1]$, where $P_{i}$ is the fraction of power that pair $i$ uses in the left band and $\bar{P}_{i}=1-P_{i}$ is the fraction of power in the right band. The utility function (payoff) that each player aims at maximizing is defined as the sum achievable Shannon rate over the two bands when the interference from the other players is treated as noise.

The purpose of this initial analysis is to investigate how the topology of the network influences the strategic split of transmission power across channels. First, we investigate the performance in terms of sum rate achieved by the network when the spectrum is split into two channels and the split of transmission powers across channels constitutes a Nash equilibrium. We also compare this with the solution achieved under the optimal transmission power split. Second, we investigate the dependence of the Nash and optimal transmission power splits on the network topology. We find that the answers to both these questions are subtle, even for the simplest non-trivial case of two channels. Extensions to larger numbers of channels are likewise of interest, but we leave investigation of these questions for more than two channels as future work.

We assume a channel model with pure pathloss attenuation, where $\alpha$ is the pathloss exponent. Also, let $\eta$ be the noise power on each band. First, we introduce the game in which the utility function of the players is given by the sum of the Shannon rate of the two bands, considering as interference the sum over all the other transmitting nodes. We refer to this game as $\Sigma$-interference game and 


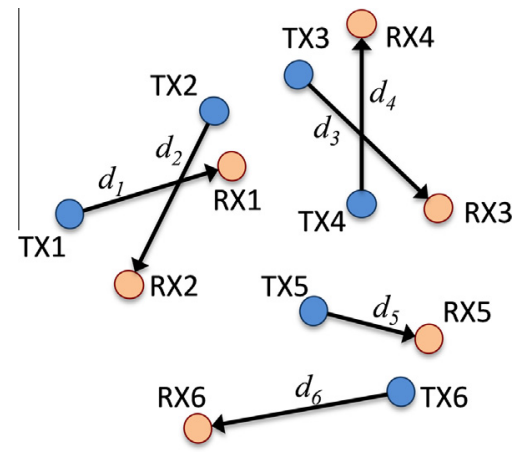

Fig. 1. Illustration of some of the Tx-Rx pairs, where the Tx locations form a BPP, and the Rx locations are selected to sit uniformly at random at a fixed distance $d_{i}$ from the associated Tx.

the utility function of the generic player $i$ for transmission powers $P \equiv\left(P_{1} \ldots P_{N}\right) \in[0,1]^{N}$ is:

$$
\begin{aligned}
U_{i}^{\Sigma}(P)= & \log _{2}\left(1+\frac{P_{i} d_{i}^{-\alpha}}{\eta+\sum_{j \neq i} P_{j} x_{i j}^{-\alpha}}\right) \\
& +\log _{2}\left(1+\frac{\bar{P}_{i} d_{i}^{-\alpha}}{\eta+\sum_{j \neq i} \bar{P}_{j} x_{i j}^{-\alpha}}\right),
\end{aligned}
$$

where $x_{i j} \equiv\left\|u_{j}-v_{i}\right\|$ is the distance between receiver $v_{i}$ and transmitter $\mathrm{u}_{j}$.

Similarly, we define the M-interference game. In this case, the interference sum is approximated by its maximum value. In other words, each transmitter makes a decision on the best strategy based on the nearest interferer only. Formally, players and actions are the same as defined above, but the utility function of the generic player $i$ for transmission powers $P \equiv\left(P_{1} \ldots P_{N}\right) \in[0,1]^{N}$ is:

$$
\begin{aligned}
U_{i}^{\mathrm{M}}(P)= & \log _{2}\left(1+\frac{P_{i} d_{i}^{-\alpha}}{\eta+\max _{j \neq i} P_{j} x_{i j}^{-\alpha}}\right) \\
& +\log _{2}\left(1+\frac{\bar{P}_{i} d_{i}^{-\alpha}}{\eta+\max _{j \neq i} \bar{P}_{j} x_{i j}^{-\alpha}}\right) .
\end{aligned}
$$

Introducing the $\mathrm{M}$-interference game allows us to have a more tractable model. Furthermore, the relationship between the two games can be formally discussed. In particular, the sum and maximum (shot noise) interference processes seen at receiver $v_{j}$ under BPP $\Pi_{N}$ are:

$\Sigma_{\Pi_{N}}\left(v_{j}\right) \equiv \sum_{u_{i} \in \Pi_{N} \backslash\left\{u_{j}\right\}}\left\|u_{i}-v_{j}\right\|^{-\alpha}$

$\mathrm{M}_{\Pi_{N}}\left(\mathrm{v}_{j}\right) \equiv \max _{u_{i} \in \Pi_{N} \backslash\left\{u_{j}\right\}}\left\|u_{i}-v_{j}\right\|^{-\alpha}$.

Properties of these processes as well as their relationship have been widely discussed in the literature on probability. As highlighted in [26] (Section 2.5), the sum interference RV $\Sigma$ is subexponential, meaning that:

$\lim _{z \rightarrow \infty} \frac{\mathbb{P}\left(\Sigma_{\Pi_{N}}\left(v_{j}\right)>z\right)}{\mathbb{P}\left(M_{\Pi_{N}}\left(v_{j}\right)>z\right)}=1$.

This shows that the two processes have Complementary Cumulative Distribution Functions (CCDFs) that are asymptotically equal. In other words, the probability of the sum being large is roughly the same as the probability of the max being large. As pointed out in [26], large sums occur due to a small number of large individual contributions; they do not occur due to a large number of small individual contributions.

Furthermore, as discussed in [27], one can define a homogeneous marked point process (with marks representing, in our context, transmission power), with an intensity thinned by retaining those points for which the sum $(\max )$ interference seen within a certain radius of each point is below a specified threshold. Analogous to (1), the ratio of these intensities from thinning using a sum vs. max interference representation has an asymptotic limit (in lambda), which is a function of the pathloss exponent alpha (c.f. Section 4.4 in [27]). Furthermore, this ratio approaches one as $\alpha \rightarrow \infty$.

The aforementioned analysis suggests that we can approximate the $\Sigma$-interference game with the more tractable M-interference game. The "quality" of this approximation is discussed throughout the paper.

\section{The 2-player game}

In this section, we consider the spectrum sharing game when $N=2$. The reference topology is reported in Fig. 2 . We label the distance between the two transmitters $t$ and, assuming that the corresponding receivers are placed uniformly around each transmitter $i$ at a distance $d_{i}, a$ and $b$ are uniform angles in the range $[0,2 \pi]$. For simplicity, we call $x_{1}$ and $x_{2}$ the distance between receiver 1 and transmitter 2 and the distance between receiver 2 and transmitter 1 , respectively. In this particular case, the $\Sigma$ interference game and $\mathbf{M}$-interference game coincide and the utility function of player 1 for transmission powers $P \equiv\left(P_{1}, P_{2}\right) \in[0,1]^{2}$ is

$U_{1}(P)=\log _{2}\left(1+\frac{P_{1} d_{1}^{-\alpha}}{\eta+P_{2} X_{1}^{-\alpha}}\right)+\log _{2}\left(1+\frac{\overline{P_{1}} d_{1}^{-\alpha}}{\eta+\bar{P}_{2} X_{1}^{-\alpha}}\right)$

and for player 2 is

$U_{2}(P)=\log _{2}\left(1+\frac{P_{2} d_{2}^{-\alpha}}{\eta+P_{1} x_{2}^{-\alpha}}\right)+\log _{2}\left(1+\frac{\bar{P}_{2} d_{2}^{-\alpha}}{\eta+\bar{P}_{1} x_{2}^{-\alpha}}\right)$.

First, we characterize the game deriving pure strategy Nash equilibria and optimal solution when the topology is assumed to be fix. Then, we provide a stochastic charac-

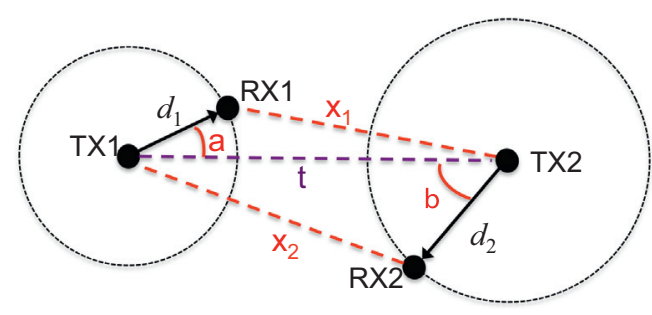

Fig. 2. The considered 2-player topology, where the two transmitters are randomly placed and $t$ is the distance between them. Receivers are also deployed randomly at distance $d_{1}$ and $d_{2}$ from the transmitters. 
terization of the game assuming that nodes are randomly deployed over a given area.

\subsection{Pure strategy Nash equilibria and optimal solution}

Each one of the two players is assumed to be selfish, that is, it allocates power between the two bands trying to maximize the achieved throughput (i.e., without taking into account the global optimum). Therefore, the stable operating points for the two players are the Nash equilibria. A Nash equilibrium is a pair $\left(P_{1}, P_{2}\right) \in[0,1]^{2}$ from which neither player has incentive to unilaterally deviate. First, recall that the best response for player $1, P_{1}^{*}\left(P_{2}\right)$, can be easily derived setting equal to zero the partial derivative with respect to $P_{1}$ of Eq. (2) and is

$P_{1}^{*}\left(P_{2}\right)=\left[\frac{1}{2}+\frac{x_{1}^{-\alpha}}{d_{1}^{-\alpha}}\left(\frac{1}{2}-P_{2}\right)\right]_{0}^{1}$,

where we define $[x]_{0}^{1}:=\max (0, \min (1, x))$. The best response for player $2, P_{2}^{*}\left(P_{1}\right)$, can be similarly derived. Then, the Nash equilibria are the points in which best responses of different players intersect. We can now state the following.

Theorem 4.1. The 2-player game has Nash equilibria given by

$\mathrm{NE}= \begin{cases}\{(0.5,0.5),(0,1),(1,0)\} & \text { if } x_{1} \leqslant d_{1}, x_{2} \leqslant d_{2}, \\ \left\{(0.5,0.5),\left(\beta_{1}^{+}, 0\right),\left(\beta_{1}^{-}, 1\right)\right\} & \text { if } x_{1}>d_{1}, x_{1} x_{2}<d_{1} d_{2}, \\ \left\{(0.5,0.5),\left(0, \beta_{2}^{+}\right),\left(1, \beta_{2}^{-}\right)\right\} & \text {if } x_{2}>d_{2}, x_{1} x_{2}<d_{1} d_{2}, \\ \left\{\left(P_{1}, P_{2}\right) \in \mathcal{L}\right\} & \text { if } x_{1} x_{2}=d_{1} d_{2} \\ \{(0.5,0.5)\} & \text { if } x_{1} x_{2}>d_{1} d_{2},\end{cases}$

where $\quad \beta_{i}^{ \pm}=0.5\left(1 \pm x_{i}^{-\alpha} / d_{i}^{-\alpha}\right) \quad$ and $\quad \mathcal{L}=\left\{\left(P_{1}, P_{2}\right): P_{1}=\right.$ $\left.0.5+x_{1}^{-\alpha} / d_{1}^{-\alpha}\left(0.5-P_{2}\right) \mid x_{1} x_{2}=d_{1} d_{2}\right\}$.

Proof. By definition, Nash equilibria are the point in which the two best responses cross. Then, substituting Eq. (4) into the equation $P_{1}^{*}\left(P_{2}\right)=P_{2}^{*}\left(P_{1}\right)$, one can derive the set of NE given in Eq. (5). (Refer to [28], Section 6.4.2, for the complete proof.)

Since, in general, the Nash equilibrium reflects the selfish behavior of the players, it is often inefficient from the system point of view. Therefore, it is useful to compare the Nash equilibria of a game with the "globally" optimal solution, i.e., the one that could be achieved with a centralized control. Usually, this comparison is done in terms of social utility, e.g., the sum of the utility of all the players, $U_{T}=U_{1}+U_{2}$. In particular, the social/global optimal sum utility is

$U_{T}^{*}=\max _{\left(P_{1}, P_{2}\right) \in[0,1]^{2}} U_{T}\left(P_{1}, P_{2}\right)$.

This quantity exists by virtue of the fact that $U_{T}$ is continuous and bounded on $[0,1]^{2}$. Unfortunately, providing explicit characterization of such optimal solution is possible only for the symmetric case $x_{1}=x_{2}$ and $d_{1}=d_{2}$. However, extensive analysis has shown that in most of the cases the following claim, which is valid for general $x_{1}$ and $x_{2}$, assuming $d_{1}=d_{2}$, holds.

Claim 4.1. The optimal solution for the 2-player game, when $d_{1}=d_{2}=d$, is given by

Opt $= \begin{cases}\{(0,1),(1,0)\} & \text { if } f_{o p t}<0 \\ \left\{\left(P_{i}, P_{j}\right): P_{i}+P_{j}=1\right\} & \text { if } f_{o p t}=0 \\ \{(0.5,0.5)\} & \text { if } f_{o p t}>0\end{cases}$

where $f_{\text {opt }}=x_{1}^{-\alpha} x_{2}^{-\alpha}+\eta x_{1}^{-\alpha}+\eta x_{2}^{-\alpha}-\eta d^{-\alpha}$. Moreover, the function $U_{T}$ is concave for $f_{\text {opt }}>0$, and has a saddle point at $P=(0.5,0.5)$ for $f_{\text {opt }}<0$.

Proof. As mentioned above, a formal proof is provided only for the symmetric case $x_{1}=x_{2}$ (cfr. Appendix D in [28]). Furthermore, to extend such result, we have studied the Karush-Kuhn-Tucker (KKT) conditions for the optimization problem. The analysis shows that $f_{\text {opt }}<0$ is a necessary conditions for $(0,1)$ and $(1,0)$ to be local optima. Extensive numerical analysis supports our claim for the general case $x_{1} \neq x_{2}$. (Refer to [28], Section 6.3.2, for the complete analysis.) $\square$

The "quality" of an equilibrium can be assessed using the concepts of Price of Stability (PoS) [29] and Price of Anarchy (PoA) [30]. They are, respectively, the ratio between the optimal solution that could be achieved by players in a centralized system and the best/worst Nash equilibrium. Namely, for $\mathrm{NE} \subseteq[0,1]^{2}$ the set of Nash equilibria, we have:

$\begin{aligned} \mathrm{PoS} & =\frac{U_{T}^{*}}{\max _{\left(P_{1}, P_{2}\right) \in \mathrm{NE}} U_{T}\left(P_{1}, P_{2}\right)}, \\ \mathrm{PoA} & =\frac{U_{T}^{*}}{\min _{\left(P_{1}, P_{2}\right) \in \mathrm{NE}} U_{T}\left(P_{1}, P_{2}\right)} .\end{aligned}$

The comprehensive characterization of the game is reported in Fig. 3, assuming $d_{1}=d_{2}=d$. Note that there exist four regions. When $x_{1} x_{2}<d^{2}$, the game admits three equilibria and the optimum is in $(0,1)$ and $(1,0)$. In particular, when both $x_{1}$ and $x_{2}$ are smaller than $d$, the PoS is one, since the best equilibrium and the optimum coincide. In contrast, when $x_{1}$ (or $x_{2}$ ) is greater than $d$, the best equilibrium is worse than the optimum, then the PoS is greater than one. The PoA is in both cases greater than one. When $x_{1} x_{2}>d^{2}$ and $f_{\text {opt }}<0$ the game admits a unique equilibrium, that does not coincide with the optimum, then PoS and PoA coincide and are greater than one. In contrast, when $f_{\text {opt }}>0$ the optimal solution and the unique equilibrium coincide. Furthermore, along the curve $x_{1} x_{2}=d^{2}$ the two best responses coincide and there exists an infinite number of equilibria.

Finally, the quality of Nash equilibria can also be characterized by their stability. A Nash equilibrium is stable if a small change in strategies for one player leads to a situation where the player with the small change in his strategy will return immediately to the Nash 


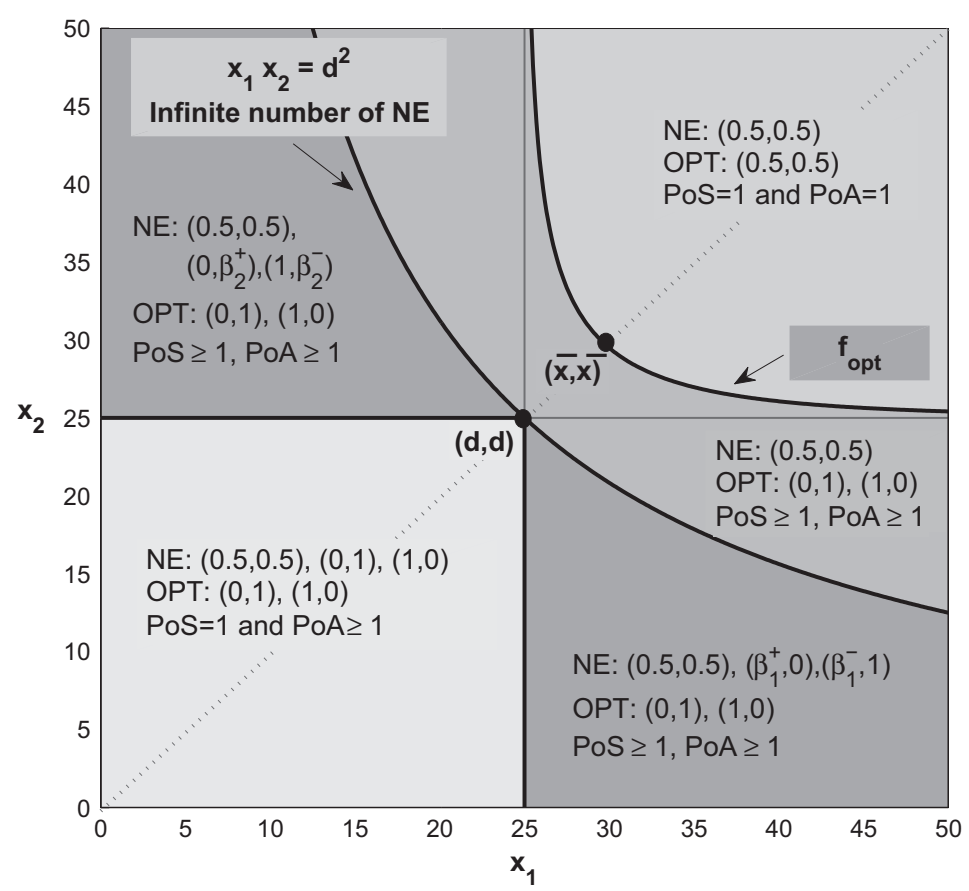

Fig. 3. Nash equilibria, optimal allocations, PoS and PoA for the 2-player game as a function of $x_{1}$ and $x_{2}$, when $d_{1}=d_{2}=d=25$.

equilibrium. We now prove the following theorem regarding the stability of the equilibria.

Theorem 4.2. The Nash equilibria of the 2-player game can be classified as follows:

- When $x_{1} x_{2}>d_{1} d_{2},(0.5,0.5)$ is the unique equilibrium and is stable.

- When $x_{1} x_{2}=d_{1} d_{2}$, there is an infinite number, $\forall\left(P_{i}, P_{j}\right) \in \mathcal{L}$, of unstable equilibria.

- When $x_{1} x_{2}<d_{1} d_{2}$, there are 3 equilibria and $(0.5,0.5)$ is the only unstable equilibrium.

Proof. To prove the theorem, let us assume that the players are at the equilibrium in $(0.5,0.5)$ and one of the two decides to deviate of a small $\epsilon$. Let us observe the sequence of the best responses:

$P_{2}=\frac{1}{2}+\epsilon \Rightarrow P_{1}^{*}\left(P_{2}\right)=\frac{1}{2}+\frac{x_{1}^{-\alpha}}{d_{1}^{-\alpha}}\left(\frac{1}{2}-\frac{1}{2}-\epsilon\right)$,

$P_{1}=\frac{1}{2}-\frac{x_{1}^{-\alpha}}{d_{1}^{-\alpha}} \epsilon \Rightarrow P_{2}\left(P_{1}\right)=\frac{1}{2}+\frac{x_{2}^{-\alpha}}{d_{2}^{-\alpha}}\left(\frac{1}{2}-\frac{1}{2}+\frac{x_{1}^{-\alpha}}{d_{1}^{-\alpha}} \epsilon\right)$.

After $N$ cycles, we obtain:

$$
\begin{aligned}
& P_{1}=\frac{1}{2}-\frac{x_{1}^{-\alpha}}{d_{1}^{-\alpha}}\left(\frac{x_{1}^{-\alpha} x_{2}^{-\alpha}}{d_{1}^{-\alpha} d_{2}^{-\alpha}}\right)^{N} \epsilon=\frac{1}{2}-\frac{x_{1}^{-\alpha}}{d_{1}^{-\alpha}}\left(\frac{d_{1} d_{2}}{x_{1} x_{2}}\right)^{\alpha N} \epsilon, \\
& P_{2}=\frac{1}{2}+\left(\frac{x_{1}^{-\alpha} x_{2}^{-\alpha}}{d_{1}^{-\alpha} d_{2}^{-\alpha}}\right)^{N} \epsilon=\frac{1}{2}+\left(\frac{d_{1} d_{2}}{x_{1} x_{2}}\right)^{\alpha N} \epsilon .
\end{aligned}
$$

Therefore, if $x_{1} x_{2}>d_{1} d_{2}$ the two best responses converge for $N \rightarrow \infty$ to $(0.5,0.5)$, proving the first part of the theorem. In contrast, when $x_{1} x_{2}<d_{1} d_{2}$ the equilibrium in $(0.5)$ is not stable.

We now prove that the other two equilibria, which exist when $x_{1} x_{2}<d_{1} d_{2}$, are stable. Consider the case in which $x_{1}<d_{1}$ and $x_{2}<d_{2}$. Assume that the users have reached the equilibrium in $(0,1)$. Note that player 1 has a best response equal to 0 for every $P_{2} \in\left[0.5+0.5 d_{1}^{-\alpha} / x_{1}^{-\alpha}, 1\right]$. This means that if player 2 deviates of an $\epsilon<0.5 d_{1}^{-\alpha} / x_{1}^{-\alpha}$, the best response of player 1 does not change, and therefore also player 2 will be incentivized to return to the equilibrium $(0,1)$, since her best response is equal to 1 for every $P_{1} \in\left[0,0.5-0.5 d_{2}^{-\alpha} / x_{2}^{-\alpha}\right]$.

Similar considerations can be derived when $x_{1} x_{2}<d_{1} d_{2}$ but either $x_{1}>d_{1}$ or $x_{2}>d_{2}$. In particular one can observe that the best response dynamics push the users in a point different from the equilibrium, but at the next step they will come back again at the same stable point.

Finally, when $x_{1} x_{2}=d_{1} d_{2}$ all the equilibria (infinite number) are unstable. In fact, every $\epsilon$ deviation in the played strategies leads the two player to a new different equilibrium.

From this analysis, we can conclude that the equilibrium (as well as the corresponding PoS/PoA) and its stability depend upon the two interference distances $\left(x_{1}, x_{2}\right)$. When node positions are random, it follows that distances are random, and thus the equilibrium is random. Therefore, we now provide a stochastic characterization of the game. 


\subsection{Stochastic analysis of the 2-player game}

\subsubsection{Conditional joint pdf of the interference distances}

We refer to Fig. 2 and we assume that the two transmitters are placed at random and we condition on the distance separating them $t$. Note that angles $\mathrm{a}$ and $\mathrm{b}$ are independent random variables. It follows that the interference distances $x_{1}, x_{2}$ are conditionally independent given $t$ :

$$
\begin{aligned}
F_{\mathrm{x}_{1}, \mathrm{x}_{2}} \mid \mathrm{t}\left(x_{1}, x_{2} \mid t\right) & \equiv \mathbb{P}\left(\mathrm{x}_{1} \leqslant x_{1}, \mathrm{x}_{2} \leqslant x_{2} \mid t\right) \\
& =\mathbb{P}\left(\mathrm{x}_{1} \leqslant x_{1} \mid t\right) \mathbb{P}\left(\mathrm{x}_{2} \leqslant x_{2} \mid t\right) .
\end{aligned}
$$

Using the law of cosines $x_{1}^{2}=d_{1}^{2}+t^{2}-2 d_{1} t \cos a$ :

$$
\begin{aligned}
\mathbb{P}\left(\mathrm{x}_{1} \leqslant x_{1} \mid t\right) & =\mathbb{P}\left(d_{1}^{2}+t^{2}-2 d_{1} t \cos \mathrm{a} \leqslant x_{1}^{2} \mid t\right) \\
& =\mathbb{P}\left(\cos \mathrm{a} \geqslant \frac{\left(d_{1}^{2}+t^{2}\right)-x_{1}^{2}}{2 d_{1} t} \mid t\right) .
\end{aligned}
$$

The following proposition is elementary.

Proposition 4.3. The random variable $\mathrm{w}$, cosine of a uniformly distributed angle, has the following distribution:

$$
f_{w}(w)=\frac{1}{\pi \sqrt{1-w^{2}}}, F_{w}(w)=\frac{1}{2}+\frac{1}{\pi} \sin ^{-1} w, \quad w \in[-1,1] .
$$

Using this result, we can now state the following theorem.

Theorem 4.4. The joint pdf of $\mathrm{x}_{1}, \mathrm{x}_{2}$ conditioned on $t$ is given by

$$
\begin{aligned}
& f_{x_{1}, x_{2}} \mid t\left(x_{1}, x_{2} \mid t\right)= \begin{cases}\frac{x_{1} x_{2}}{\pi^{2} d_{1} d_{2} b(t)} & \left|d_{1}-t\right| \leqslant x_{1} \leqslant d_{1}+t \\
0 & \text { and }\left|d_{2}-t\right| \leqslant x_{2} \leqslant d_{2}+t \\
\text { else }\end{cases} \\
& \text { and } b(t)=t^{2} \sqrt{\left[1-\left(\frac{d_{1}^{2}+t^{2}-x_{1}^{2}}{2 d_{1} t}\right)^{2}\right]\left[1-\left(\frac{d_{2}^{2}+t^{2}-x_{2}^{2}}{2 d_{2} t}\right)^{2}\right]} .
\end{aligned}
$$

Proof. For the sake of brevity, we only report some mathematical steps. From Eq. (10) and Proposition 4.3:

$$
\mathbb{P}\left(\cos \mathrm{a} \geqslant \frac{\left(d_{1}^{2}+t^{2}\right)-x_{1}^{2}}{2 d_{1} t} \mid t\right)=\bar{F}_{\mathrm{w}}\left(\frac{\left(d_{1}^{2}+t^{2}\right)-x_{1}^{2}}{2 d_{1} t} \mid t\right) .
$$

Then $F_{\mathrm{x}_{1}, \mathrm{x}_{2}} \mid \mathrm{t}\left(x_{1}, x_{2} \mid t\right)$ follows by Eq. (9), requiring the argument of the cdf $F_{\mathrm{w}}(w)$ to be between -1 and 1 . Eq. (11) follows directly from taking the double partial derivative with respect to $x_{1}$ and $x_{2}$ :

$f_{\mathrm{x}_{1}, \mathrm{x}_{2}} \mid \mathrm{t}\left(x_{1}, x_{2} \mid t\right)=\frac{\partial^{2}}{\partial x_{1} \partial x_{2}} F_{\mathrm{x}_{1}, \mathrm{x}_{2} \mid \mathrm{t}}\left(x_{1}, x_{2} \mid t\right)$.

Fig. 4 shows the conditional joint pdf and cdf when $d_{1}=d_{2}=1$ and $t=3$. Note that we obtain a function different from zero only in the region $\left\{\left(x_{1}, x_{2}\right):\left|d_{1}-t\right| \leqslant x_{1}\right.$ $\left.\leqslant d_{1}+t,\left|d_{2}-t\right| \leqslant x_{2} \leqslant d_{2}+t\right\}$, consistent with the topology shown in Fig. 2. Indeed, due to the geometry of the problem (the sum of any two sides of a triangle must be greater than the third side), the following inequalities must hold:

$$
\begin{aligned}
& \left|d_{1}-t\right| \leqslant x_{1} \leqslant d_{1}+t \\
& \left|d_{2}-t\right| \leqslant x_{2} \leqslant d_{2}+t \\
& \max \left\{\left|d_{1}-x_{1}\right|,\left|d_{2}-x_{2}\right|\right\} \leqslant t \leqslant \min \left\{d_{1}+x_{1}, d_{2}+x_{2}\right\}
\end{aligned}
$$

\subsubsection{Joint pdf of the mutual interference distances}

For any distribution on the random distance $t$ separating the transmitters, we obtain the joint (unconditioned) distribution for $\left(x_{1}, x_{2}\right)$ by the total probability theorem:

$f_{\mathrm{x}_{1}, \mathrm{x}_{2}}\left(x_{1}, x_{2}\right)=\int_{0}^{\infty} f_{\mathrm{x}_{1}, \mathrm{x}_{2} \mid \mathrm{t}}\left(x_{1}, x_{2} \mid t\right) f_{\mathrm{t}}(t) \mathrm{d} t$.

Note that, as shown in Eq. (12), the RVs $x_{1}, x_{2}$ are not (unconditionally) independent.

We will henceforth assume the random transmitter separation distance $t$ is determined by placing one of the transmitters at the origin of a disk of radius $L$, and placing the second transmitter uniformly at random in the disk. We emphasize, however, that Eq. (13) holds for any distribution on $t$, and our assumption is merely for the purpose of concreteness. Under this assumption, the RV t has cdf and pdf:
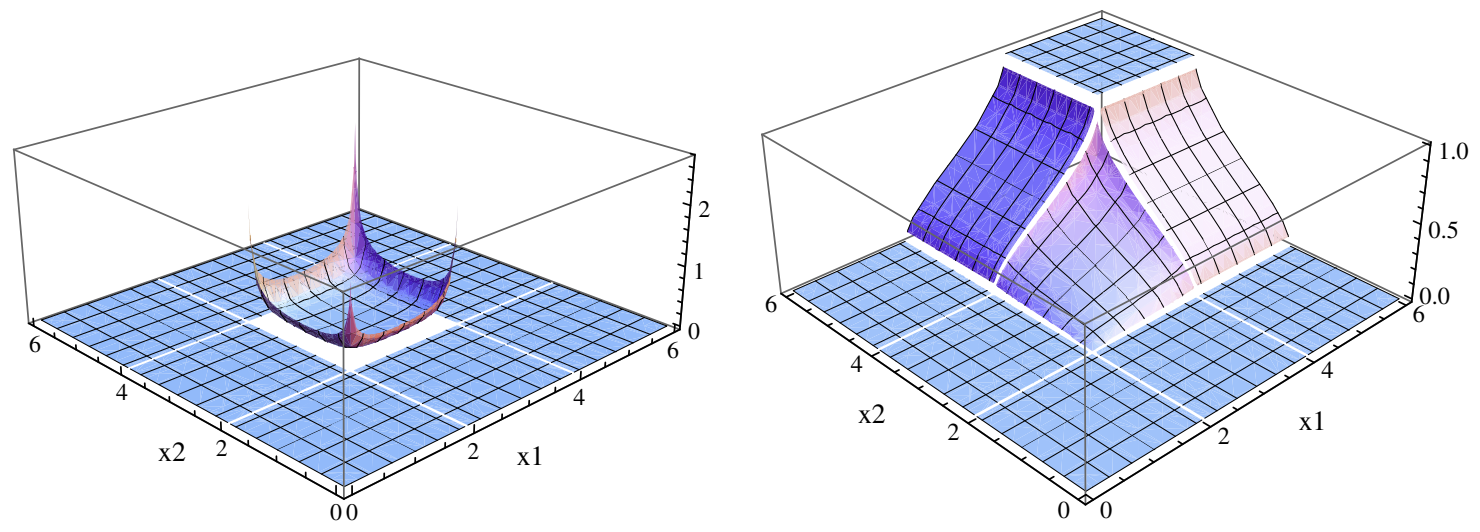

Fig. 4. Conditional joint pdf (left) and cdf (right) of $\mathrm{x}_{1}$ and $\mathrm{x}_{2}$ when $d=1$ and $t=3$. 
Table 1

Different events that are considered in the stochastic analysis of the game when $d_{1}=d_{2}=d$.

\begin{tabular}{ll}
\hline "event" & $\mathcal{P}$ \\
\hline unique & $x_{1} x_{2}>d^{2}$ \\
$(0.5,0.5)-(0,1)-(1,0)$ & $x_{1}<d \wedge x_{2}<d$ \\
mixed & $x_{1} x_{2}<d^{2} \wedge\left(x_{1}>d \vee x_{2}>d\right)$ \\
infinite & $x_{1} x_{2}=d^{2}$ \\
coincide w/opt & $\left(x_{1} x_{2}\right)^{-\alpha}+\eta\left(x_{1}^{-\alpha}+x_{2}^{-\alpha}-d^{-\alpha}\right)>0$ \\
\hline
\end{tabular}

$F_{\mathrm{t}}(t)=\left(\frac{t}{L}\right)^{2}, f_{\mathrm{t}}(t)=\frac{2 t}{L^{2}}, 0 \leqslant t \leqslant L$.

We can now state the following theorem.

Theorem 4.5. Suppose the distance $\mathrm{t}$ between the two transmitters has the distribution in Eq. (14). Then the joint pdf of $\mathrm{x}_{1}, \mathrm{x}_{2}$ is given by

$f_{\mathrm{x}_{1}, \mathrm{x}_{2}}\left(x_{1}, x_{2}\right)=\frac{2 x_{1} x_{2}}{\pi^{2} d_{1} d_{2} L^{2}} \int_{\max \left\{\left|d_{1}-x_{1}\right|,\left|d_{2}-x_{2}\right|\right\}}^{\min \left\{d_{1}+x_{1}, d_{2}+x_{2}\right\}} \frac{t}{b(t)} \mathrm{d} t$,

where $b(t)$ is given in Theorem 4.4 and the support of $\left(\mathrm{x}_{1}, \mathrm{x}_{2}\right)$ is given by Eq. (12).

Proof. Substitute Eq. (14) for $f_{\mathrm{t}}(t)$ and Eq. (11) for $f_{\mathrm{x}_{1}, \mathrm{x}_{2} \mid \mathrm{t}}\left(x_{1}, x_{2} \mid t\right)$ in Eq. (13), using constraints in Eq. (12).

Note that, in general, it is not possible to provide close form expression of the integral in Eq. (15). Numerical evaluation, however, is straightforward.

\subsubsection{Distribution on the Nash equilibria}

We can now use the joint distribution in Theorem 4.5 to compute the distribution of the equilibria in Fig. 3. Each equilibria's probability can be evaluated via:

$\int_{\mathcal{P}} f_{x_{1}, x_{2}}\left(x_{1}, x_{2}\right) d x_{1} d x_{2}$

where $\mathcal{P}$ is the set of points that defines that region. Table 1 summaries the different cases, which are also illustrated in Fig. 5. Note that due to the restriction adopted in Claim 4.1, we assume hereafter in this section $d_{1}=d_{2}=d$, so that we can include the comparison with the optimal solution. In particular, unique refers to the probability that the equilibrium is unique. When the equilibrium is not unique, we consider two cases. The case in which the three equilibria are $(0.5,0.5),(0,1)$ and $(1,0)$, i.e., $(0.5,0.5)-(0,1)-(1,0)$, and the case in which the equilibria depend on the parameter $\beta^{ \pm}$, (mixed). For completeness, we also provide the probability that there is an infinite number of equilibria (infinite). Finally, we evaluate the probability that the unique equilibrium and the optimal solution coincide (coincide w/opt).

Numerical results for all these probabilities are reported in Fig. 6. We consider different values of $d$, with $L$ varying from 0.1 to 50 . We assume $\eta=10^{-3}$ and $\alpha=4$.

The probability of having a unique equilibrium, that corresponds to the case in which players decide to use the whole spectrum, increases with $L$ and approaches 1 when $L \simeq 5 d$. This is reasonable since interference decreases with $L$. The probability that the optimum and the unique equilibrium coincide has a similar behavior of the probability of uniqueness, but it is lower. This comes from the fact that when the equilibrium and the optimum coincide, the equilibrium is also unique, but the opposite is not always true. Fig. 7 (left) reports the topology when $L \rightarrow 0$, i.e., $L=\epsilon$, for any small $\epsilon>0$. In this case, each $x$ can be a little bit greater or a little bit smaller than $d$ (remember that $x^{2}=d^{2}+\epsilon^{2}-2 d \epsilon \cos a$ ). Fig. 7 (right) shows why, when $L$ approaches zero, the probability of unique equilibrium is equal to 0.5 (given by the probability that both the $x$ s are greater than $d$ ). Complementary, the probability of having $\{(0,1),(1,0),(0.5,0.5)\}$ (both $x$ 's are less than $d$ ) and the mixed case (when only one $x$ is greater than $d$, but both below the curve $x_{1} x_{2}=d^{2}$ ) are both 0.25 . Finally, note that when $L$ goes to zero the optimum never coincides with the equilibrium in $(0.5,0.5)$. In fact, when $L=\epsilon$, the two $x$ 's are both close to $d$ and they do not satisfy the condition $f_{\text {opt }}>0$.

In this section, we have fully characterized the 2-player game, both assuming a deterministic and a random topology. The analysis has highlighted important properties of such game. It always admits at least one Nash equilibrium in pure strategy and at least one of such equilibria is stable. These properties are also used in the following section. We will show how the $N$-player game can be decomposed in sub-graphs and we will propose a distributed protocol that relies on the existence and stability of the 2-player subgames.

\section{The $N$-player game}

As done for the 2-player game, we can derive the response function for the $\Sigma$-interference game as

$P_{i}^{*}\left(\mathbf{P}_{-i}\right)=\left[\frac{1}{2}+\sum_{j \neq i}\left(\frac{x_{i j}}{d_{i}}\right)^{-\alpha}\left(\frac{1}{2}-P_{j}\right)\right]_{0}^{1}$,

and for the $\mathrm{M}$-interference as

$P_{i}^{*}\left(\mathbf{P}_{-i}\right)=\left[\frac{1}{2}+\max _{j \neq i}\left(\frac{x_{i j}}{d_{i}}\right)^{-\alpha}\left(\frac{1}{2}-P_{j}\right)\right]_{0}^{1}$

where $\mathbf{P}_{-i} \equiv\left(P_{1}, \ldots, P_{i-1}, P_{i+1}, \ldots, P_{N}\right)$.

Theorem 5.1. The strategy profile $P_{i}=0.5, \forall i \in N$ is always a pure strategy Nash equilibrium for both the $\Sigma$-interference game and the $\mathrm{M}$-interference game.

Proof. The proof comes directly by observing that substituting $P_{j}=0.5$ in both Eq. (16) and Eq. (17) we obtain $P_{i}=0.5, \forall i, j$.

Therefore, even when users play considering the nearest interferer only, we can still guarantee the existence of at least on pure strategy Nash equilibria.

In the following, we start analyzing the M-interference game. The comparison between the $\Sigma$-interference game and the M-interference game is treated in Section 6. 

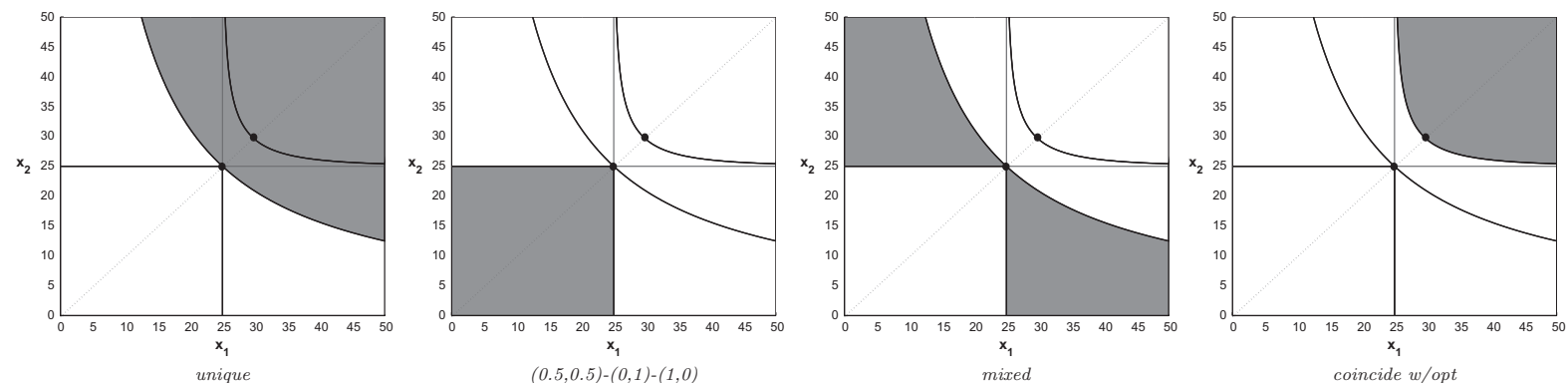

Fig. 5. Regions of Fig. 3 that we are considering in Table 1.
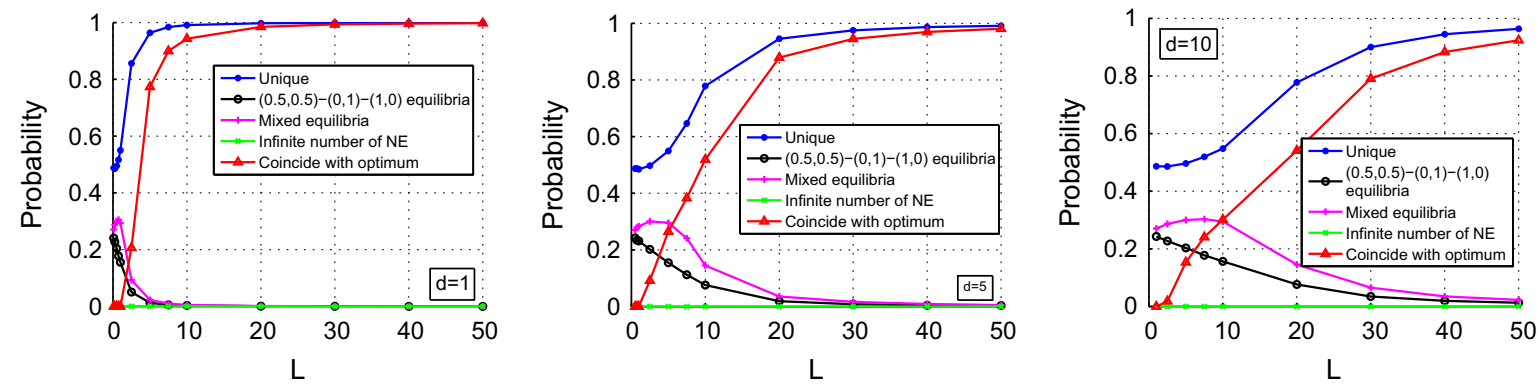

Fig. 6. Probability of having different Nash equilibria for the 2-player game, increasing $L$, for different values of $d$, assuming $\eta=10^{-3}$ and $\alpha=4$.

\subsection{Reducing the M-interference game}

In this section we show how the M-interference game allows us to reduce the $N$-player game to small subgames. We do this using a directed-influence-graph-based approach.

Definition 5.2 (Directed influence graph). The directed influence graph associated to an $\mathrm{N}$-player game is a directed graph in which each vertex corresponds to a pair of nodes, and a directed edge from $i$ to $j$ represents the fact that transmitter $u_{i}$ is the nearest interferer for receiver $\mathrm{v}_{j}$.

By definition of directed influence graph, it is worth noting that the following property holds:
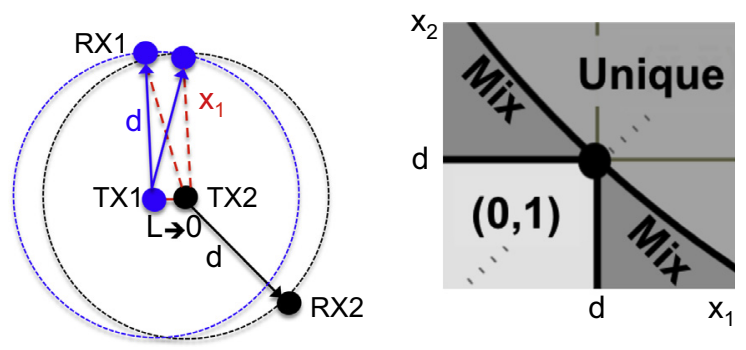

Fig. 7. When $L$ goes to zero the positions of the two transmitters coincide. The topology is shown on the left. A zoom of the corresponding region of Fig. 3 is reported on the right.
Remark 5.3. In every directed influence graph, each vertex has in-degree equal to 1 . In contrast, the out-degree might be 0,1 , or greater than 1 .

In Fig. 8, we show the same scenario reported in Fig. 1. On the left side, each receiver is associated to his closest interfering transmitter. One the right we report the associated directed influence graph. In this case, all pairs of nodes are coupled, i.e., we obtain couples of players that mutually interfere each other. Formally, we provide the following definition.

Definition 5.4 (Coupled users). Users $\left(\mathrm{u}_{i}, \mathrm{v}_{i}\right)$ and $\left(\mathrm{u}_{j}, \mathrm{v}_{j}\right)$ are coupled if the nearest interfering transmitter of receiver $v_{j}$ is $u_{i}$ and the nearest interfering transmitter of receiver $v_{i}$ is $u_{j}$.

In general, we might not have that all users are coupled. Therefore we introduce the following definitions.

Definition 5.5 (Cycle). We say that three or more pairs of users form a cycle, when the nodes of the associated directed influence graph have all in-degree and out-degree equal to 1 .

Definition 5.6 (Chain). We say that one or more pairs of users form a chain, when the nodes of the associated directed influence graph have in-degree and out-degree equal to 1 , except for the leaves of the chain that have out-degree equal to zero. 

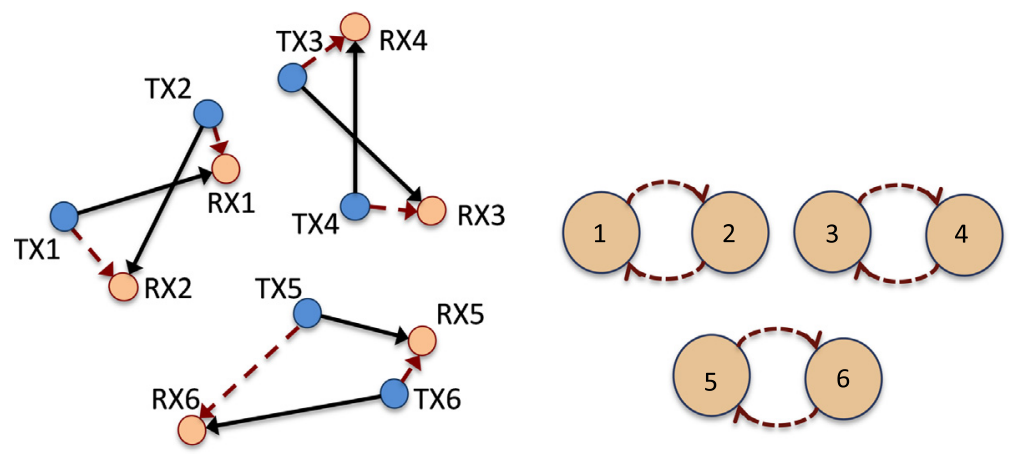

Fig. 8. The interference relationships (left) and the associated directed influence graph (right) for the 6-player game reported in Fig. 1.

Furthermore, note that every network can be represented as a directed influence graph that is composed by one or more isolated sub-graphs. In particular, every isolated subgraph has the following property.

Proposition 5.7. Every isolated sub-graph is necessarily composed by one and only one couple or cycle and might have one or more chains connected to it.

Proof. The proof proceeds by contradiction. Let us assume that one isolated sub-graphs exists with one of the following configurations: (i) two couples (or cycles), or (ii) one couple and one cycle; in both cases, couples and cycles have to be connected by a chain; then, such chain connecting couples and cycles must have by construction at least one node with in-degree equal to 2 . This contradicts Remark 5.3. Furthermore, it is easy to see that a chain cannot be isolated by itself. This comes again from Remark 5.3, i.e., each node has in-degree equal to 1 . The very same reasoning can be applied to show that isolated sub-graphs cannot exist with multiple instances of couples and cycles.

Fig. 9 reports a random topology composed by $N=20$ pairs. In Fig. 10, it is reported the associated directed influence graph. It is composed by three sub-graphs, two of them have a couple and one has a cycle. All of them have connected chains.

Finally, according to the previous classification and reminding that every node in the graph is a couple of communicating transmitter and receiver, we will adopt the following classification for the players:

\section{Coupled users}

See Definition 5.4

\section{Cycle users}

Users that belong to a cycle

Chain-of-a-couple users

Users that belong to a chain connected to coupled users Chain-of-a-cycle users

Users that belong to a chain connected to a cycle

\subsection{Coupling probability}

The reduction through directed influence graphs allows us to identify pairs of users that actually play a 2-player game. Formally, we define the probability of a typical user being coupled as:

Definition 5.8 (Coupling probability). The coupling probability $P_{c}(\lambda)$ is the probability that a typical user, say $\left(\mathrm{u}_{i}, \mathrm{v}_{i}\right)$, is coupled with the user whose transmitter, say $u_{j}$, is the
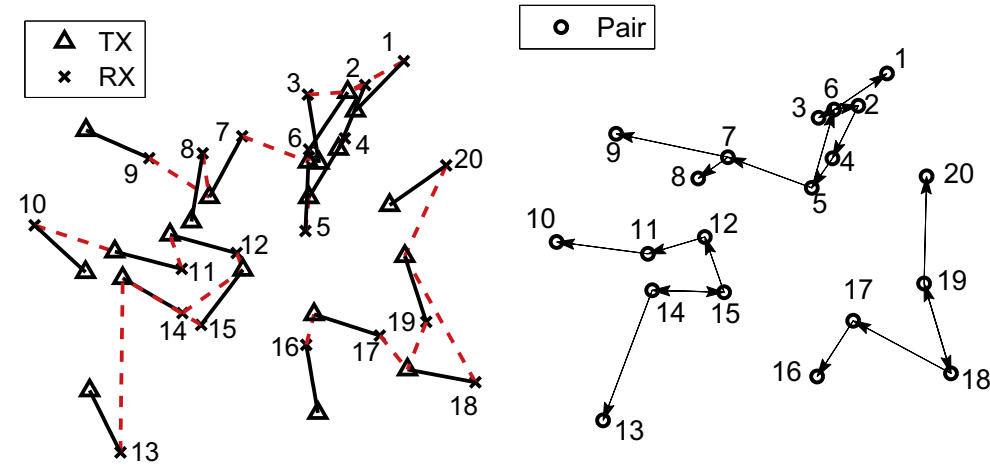

Fig. 9. (Left) Random topology composed by $N=20$ pairs, where communicating TXs and RXs are connected with a solid black line, whereas each receiver is connected to the nearest transmitter with a dotted red line. (Right) Corresponding directed influence graph. Each pairs is substituted with a circle and interference relations are marked with arrows. (For interpretation of the references to color in this figure legend, the reader is referred to the web version of this article.) 


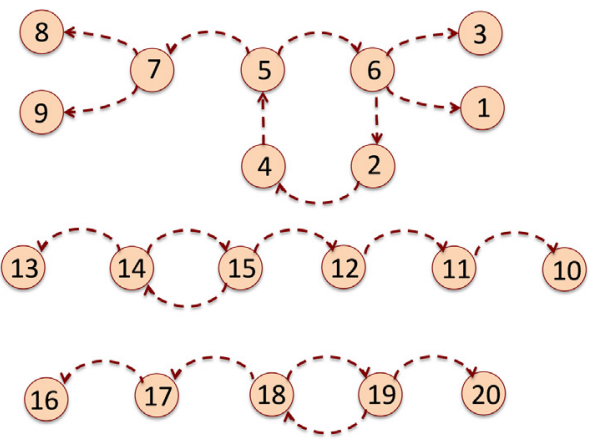

Fig. 10. Directed influence graph of the topology reported in Fig. 9. The graph is composed by three isolated sub-graphs, two of them have a couple, the other has a cycle. All of them have chains.

closest interferer to $v_{i}$, i.e., the probability that $u_{i}$ is in fact also the closest interferer to $v_{j}$ :

$P_{c}(\lambda)=\mathbb{P}\left(i=\arg \min _{k \neq j}\left\|\mathrm{u}_{k}-\mathrm{v}_{j}\right\| j=\arg \min _{k \neq i}\left\|\mathrm{u}_{k}-\mathrm{v}_{i}\right\|\right)$.

Refer to Fig. 2. Assume that $\mathrm{TX}_{2}$ is the nearest interferer for $\mathrm{RX}_{1}$. Note that this means that there is no other transmitter in the circle (say, $\mathcal{C}_{1}$ ) of radius $x_{1}$ with center $\mathrm{RX}_{1}$, i.e., $\Pi_{N} \cap \mathcal{C}_{1}=\Pi_{N}\left(\mathcal{C}_{1}\right)=0$. The probability that $\mathrm{TX}_{1}$ is the nearest interferer for $\mathrm{RX}_{2}$ is $P_{c}(\lambda)=\mathbb{P}\left(\Pi_{N}\left(\mathcal{C}_{2}\right)=\right.$ $\left.0 \mid \Pi_{N}\left(\mathcal{C}_{1}\right)=0\right)$, where $\mathcal{C}_{2}$ is the circle of radius $x_{2}$ with center $\mathrm{RX}_{2}$. Although occupancy counts of disjoint regions are dependent in a BPP, the dependence vanishes in the limit as the BPP becomes a Poisson Point Process (PPP), i.e., as $N,|\mathcal{A}| \rightarrow \infty$ with $N /|\mathcal{A}| \rightarrow \lambda$. Under this approximation we have

$P_{c}(\lambda) \approx \mathbb{P}\left(\Pi_{\lambda}(\Delta)=0\right)=\mathrm{e}^{-\lambda|\Delta|}$,

i.e., the coupling probability is approximately the void probability for a PPP $\Pi_{\lambda}$ of intensity $\lambda$ on the lune $\Delta \equiv \mathcal{C}_{2} \backslash \mathcal{C}_{1}$.

Theorem 5.9. The value for the coupling probability when $\lambda$ goes to 0 is the following constant:

$\lim _{\lambda \rightarrow 0} P_{c}(\lambda)=C_{c p} \triangleq \frac{6 \pi}{3 \sqrt{3}+8 \pi} \approx 0.6215$

Proof. In the low density (small $\lambda$ ) regime the BPP behaves like the PPP since $|\mathcal{A}|$ must be large. Recall that the average distance to a nearest neighbor in a PPP with density $\lambda$ is $1 / \sqrt{\lambda}$, and thus for small $\lambda$ the average distance is large. To obtain the value of the coupling probability in the low density regime, we can assume that distances $x_{1}$ and $x_{2}$ are both much larger than $d_{1}$ and $d_{2}$. Thus as $\lambda \rightarrow 0, d_{1}$ and $d_{2}$ can be neglected and we can assume that $x_{1}=x_{2}$. In this scenario, reported in Fig. 11, we have two circles with the same radius, whose centers are separated by a distance equal to the radius itself. Therefore, the area of the lune depends only on the parameter $x_{1}$. In particular, using the formula of the area of the lune, we obtain that in this case the area of the lune is the following:

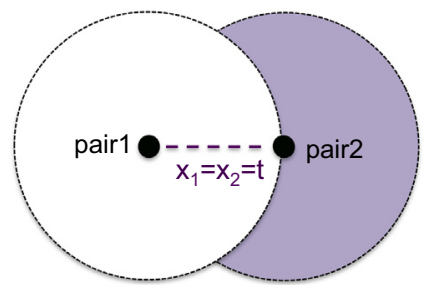

Fig. 11. Reference scenario when $\lambda$ goes to zero. Each black point represents a pair. Since the distance between pairs is very large, $d_{1}$ and $d_{2}$ can be neglected and we can assume that $x_{1}=x_{2}$.

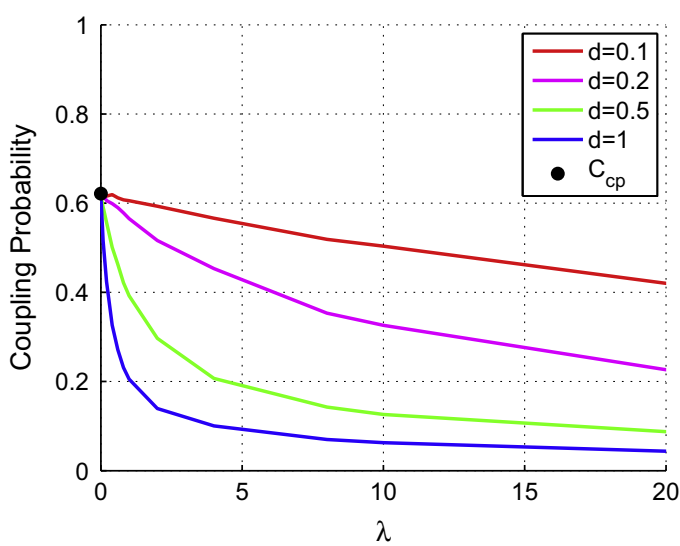

Fig. 12. Simulated coupling probability for different values of $d=d_{1}=d_{2}$ increasing $\lambda$

$\Delta=\left(\frac{\pi}{3}+\frac{\sqrt{3}}{2}\right) x_{1}^{2}$

Applying the total probability theorem:

$\left.P_{c}(\lambda)=\int_{0}^{\infty} P_{c \mid x_{1}}\left(\lambda, x_{1}\right) f_{\mathrm{x}_{1}\left(x_{1}\right.}\right) \mathrm{d} x_{1}$.

The distribution of the nearest interferer is:

$\left.f_{\mathrm{x}_{1}\left(x_{1}\right.}\right)=2 \pi \lambda x_{1} \mathrm{e}^{-\pi \lambda x_{1}^{2}} \quad x_{1} \geqslant 0$.

Substituting the expression for $f_{\mathrm{x}_{1}}\left(x_{1}\right)$ in Eq. (20) and the void probability over the area in Eq. (18), we obtain:

$P_{c}(\lambda)=\int_{0}^{\infty} \mathrm{e}^{-\lambda\left(\frac{\pi}{3}+\frac{\sqrt{3}}{2}\right) x_{1}^{2}} 2 \pi \lambda x_{1} \mathrm{e}^{-\pi \lambda x_{1}^{2}} \mathrm{~d} x_{1}=C_{c p}$.

Fig. 12 show the simulated coupling probability $P_{c}(\lambda)$ for a large range of $\lambda$, and different values of $d$, assuming $d=d_{1}=d_{2}$. The black point is $C_{c p}$.

Finally, we have numerically evaluated the probability of being of a specific type. Fig. 13 shows the probability distribution over different types of nodes increasing $\lambda$ for $d=1$ and for $d=5$. Results show that there exists a regime (the low density regime) in which nodes are either coupled or belong to a chain that is connected to a couple. Again, for 


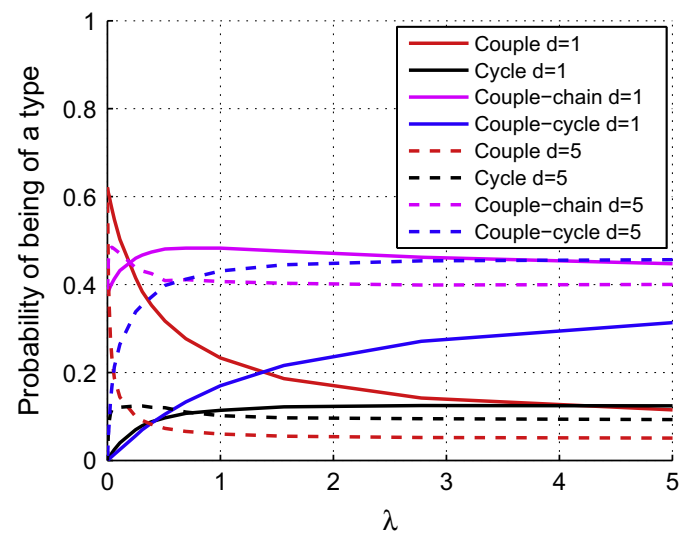

Fig. 13. Probability distribution over different types of node in the directed influence graph for $d=1$ and $d=5$, increasing $\lambda$.

$\lambda$ that goes to zero, the probability of being coupled coincide with the exact value derived in Theorem 5.9 for any value of $d$.

\subsection{Convergence through best response dynamics}

The characterization of the $\mathrm{M}$-interference game using directed influence graphs leads to scenarios with independent sub-graphs. Classification, according to Proposition 5.7, and convergence properties are reported in the following.

- Sub-graph with coupled users. In this kind of sub-graph, equilibrium can be reached using the previous analysis of the 2-player game. Namely, the coupled nodes independently reach the equilibrium based only on the mutual interfering distances. The nodes that belong to a chain reach a stable state only reacting to others' nodes choices. E.g., the node of a chain, which is closest to the couple, simply plays the best response to the choices of the nodes in the couple. The second node of the chain reacts to the choice of the first, and so on.

- Sub-graph with a cycle. Nodes in a cycle might not reach an equilibrium point following the best response dynamics. These critical cases can be managed recalling that a pure strategy equilibrium always exists, i.e., when all nodes play the strategy $(0.5,0.5)$. Therefore, nodes that belong to this type of sub-graphs can be forced to play strategy 0.5 . However, in low density regime the probability of being in a cycle goes to zero.

The analytical characterization of these sub-graphs and the study of the games played in these substructures is part of future work. In contrast, in this paper, we have shown that such decomposition exists. Furthermore, the convergence properties highlighted above will be exploited in the following section in order to propose a distributed protocol that always converges to a stable solution.

\section{Game solution algorithms}

\subsection{Best-response-based algorithm}

Having proved the existence of at least one pure strategy Nash equilibrium, we propose in this section a heuristic based on best response dynamics for both the $\Sigma$ interference game and the $\mathrm{M}$-interference game. It is worth pointing out that, although existence of Nash equilibria is certain in both cases, best response dynamics may not always converge. To this extent, the proposed best response heuristic introduces termination condition in order to guarantee the convergence to a stable point (which however is not said to be a Nash equilibrium). The idea is to let each player play the best response but only for a limited number of times, said T. Such stopping time $\mathbf{T}$ is often effective for a power control algorithm to limit the number of iterations. When a player changes its best response $\mathbf{T}$ times, then the player is forced to play 0.5 . We choose this value because we have proved that the strategy where each player plays 0.5 is always a pure strategy Nash equilibrium. The value of $\mathbf{T}$ captures the trade-off between system efficiency and system responsiveness. Roughly speaking, larger $\mathbf{T}$ may lead to better stable points (eventually equilibria), but on the other side, this requires larger convergence time. In a practical scenario of spectrum sharing, the algorithm convergence time represents a pure overhead, since the wireless nodes cannot actually use the spectrum during this phase; as an example, this may represent the time in which the competing nodes perform spectrum sensing and spectrum decision.

The Game Heuristic $(\mathrm{GH})$, reported in Algorithm 1 , implements the best response (BR) function for every player; at each cycle verifies which are the players that want to deviate and picks up randomly one of these players, applies the best response function adapting the chosen strategy and consequently evaluates again all the best responses until no player wants to deviate. Both the $\Sigma$-interference game and the $\mathrm{M}$-interference game can be implemented adapting the evaluation of the BR in Step 3 of Algorithm 1.

Algorithm 1. Game heuristic.

1: Initialize $P_{i}=\operatorname{rand}(1)$ and $T_{i}=0 \quad \forall i=1 \ldots N$

2: while There exists at least a player $k$ that has updated its $P_{k}$ in the last step do

3: Evaluate best response $B R_{i}$ for each player $i$

4: Search a player $k^{\prime}$ such that $B R_{k^{\prime}} \neq P_{k^{\prime}}$ and $T_{k^{\prime}} \leqslant \mathbf{T}$

5: if such a player exists then

6: $\quad$ if $T_{k^{\prime}}<\mathbf{T}$ then

7: $\quad P_{k^{\prime}} \leftarrow B R_{k^{\prime}}$

8: $\quad T_{k^{\prime}} \leftarrow T_{k^{\prime}}+1$

9: else

10: $\quad P_{k^{\prime}} \leftarrow 0.5$

11: $\quad T_{k^{\prime}} \leftarrow T_{k^{\prime}}+1$

12: $\quad$ end if

13: end if

14: end while 


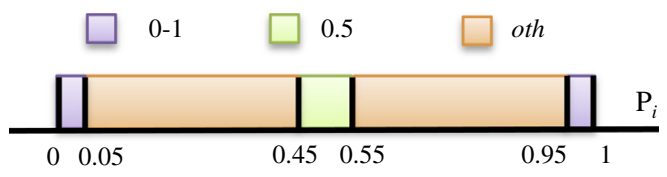

Fig. 14. Discretization of the strategy set of each player used in the simulations.

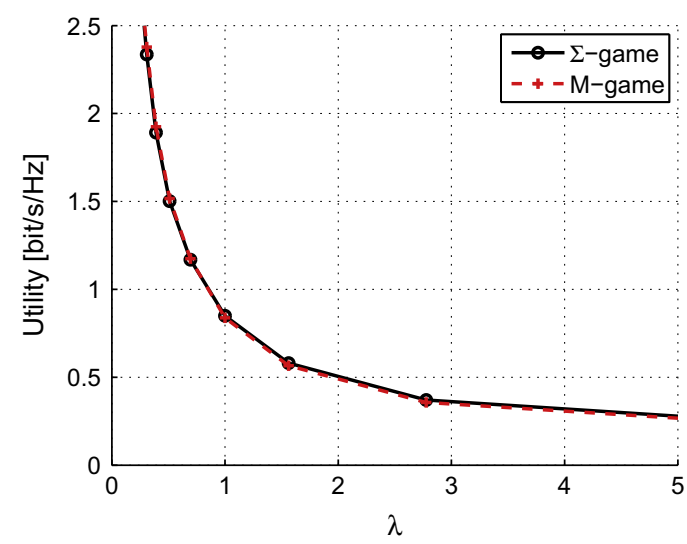

Fig. 15. Utility (Shannon rate) obtained at the $\Sigma$-equilibria and $M-$ equilibria varying $\lambda$.

We consider a scenario with $N=25$ pairs of users, at a communicating distance $d=1$. We let $\lambda$ goes from 0 to 5 (note that in order to vary $\lambda$, we vary the area $|\mathcal{A}|$ of the convex set $\mathcal{A}$ where the $N$ users are deployed). We assume $\eta=10^{-3}$ and $\alpha=4$. We fix $T=20$, and we average over $S=1000$ randomly generated instance. For each instance we derive $K=20$ different solutions (i.e., we run the game simulator/protocol starting from randomly chosen power allocation strategies and this consequently leads to different termination points). Furthermore, since the strategy set is the continuous set $[0,1]$, a discretization is needed. Also, since in the 2-player game, we have noticed that there are mainly two relevant strategies, $(0,1)-(1,0)$ and $(0.5,0.5)$, we define the strategies adopted by the users as reported in Fig. 14, where oth refers to all the strategies but $(0,1)-(1,0)$ and $(0.5,0.5)$.

We compare the different approaches in terms of utility, i.e., Shannon capacity. Numerical results show that, especially in the low density regime, the performance of the M-equilibria is close to the one that we obtain for the $\Sigma$-equilibria, in terms of both utility and fairness. ${ }^{1}$ This is reported in Fig. 15 and Fig. 16, respectively. As expected, the performance of the two heuristics are very close. The $\Sigma$-interference game leads to slightly better performance. However, it is reasonable to expect that the two heuristics lead to similar equilibria/stable points.

The problem with the heuristics is the time of convergence, as well as the probability of reaching an equilibrium point. We have pushed $\mathbf{T}$ to very large values and we still observe high probability of non convergence, thus long running time of

\footnotetext{
${ }^{1}$ We use the Jain's fairness index to measure the fairness of the throughput allocation [31].
}

the algorithm. Fig. 17 reports the probability for a user of being forced to 0.5 for different values of $\lambda$ and increasing $T$.

Therefore, although numerical results support the value of the M-interference game, we need some better way to achieve similar allocations in a distributed and fast way.

\subsection{Distributed protocol with minimal information exchange and fast convergence time}

The analysis of best response dynamics proposed in the previous section suggests that the "quality" of the points of convergence is comparable in the two games, which means that the M-game is a reasonably good approximation for the $\Sigma$-game. Building on this result, we provide a practical distributed protocol for spectrum sharing which is fast in convergence and requires minimal information exchange among wireless nodes. The pseudocode for the Distributed Sharing Protocol (DSP) that each couple of communicating nodes runs in order to decide how to allocate the power is reported in Algorithm 2.

Algorithm 2. Distributed Sharing Protocol.

1: Every transmitter $i$ starts transmitting using

2: Every transmitter sends information regarding the positions of the communicating nodes and the associated ID

3: Every pair identifies the nearest transmitter ID, and broadcast this information

4: Every node is able to evaluate if it is coupled or not

5: for every $i$ and $j$ that are coupled do

6: $i$ and $j$ play the 2-player game equilibrium, for which no coordination is required

\section{7: end for}

8: for every node is not coupled do

9: monitor of the perceived interference in the two bands

10: adapt the power allocation according to the perceived interference, i.e., play the best response

11: end for

Note that DSP requires information on the position of the neighboring nodes so that each node is able to determine either it is coupled or not. Coupled users play the best response deterministically without any best response interactions. ID information can be useful for coordination when more than one equilibrium exists (e.g., whenever the 2-player game admits as equilibrium either $(0,1)$ and $(1,0)$, the node with smaller ID plays first choosing either 0 or 1 , and the other node accordingly replies). Users that belong to a chain connected to the couple play a one-shot best response and the convergence time will depend on how long is the chain. Also in this case there is not iterative response among users. Finally, since we have shown in Section 5.3 that cycles are difficult to treat, we force users that belong to cycles to 0.5 . By construction, DSP always converge to an equilibrium of the $\mathrm{M}$-interference game 


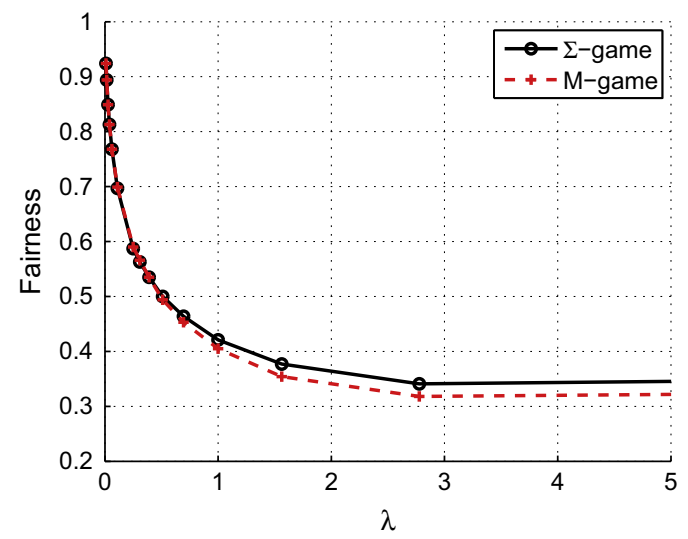

Fig. 16. Fairness obtained at the $\Sigma$-equilibria and $M$-equilibria varying $\lambda$.

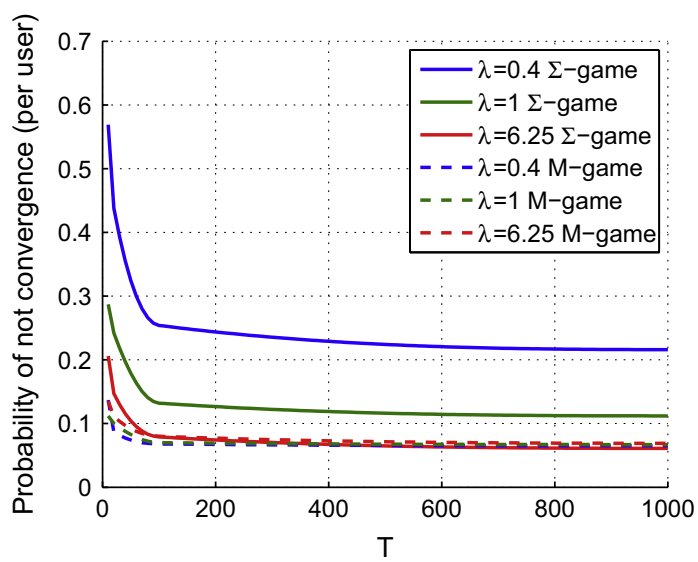

Fig. 17. Probability of being forced to termination, i.e., of not convergence, for both the $\Sigma$-equilibria and the M-equilibria and different values of $\lambda$.

Table 2

Comparison between "Game Heuristic" (GH) and "Distributed Sharing Protocol" (DSP).

\begin{tabular}{llll}
\hline & $\begin{array}{l}\text { Information } \\
\text { needed }\end{array}$ & Convergence & $\begin{array}{l}\text { Stability of the } \\
\text { reached point }\end{array}$ \\
\hline $\mathrm{GH}-$ & $\begin{array}{l}\text { Convergence is } \\
\text { guaranteed only by } \\
\text { introducing a } \\
\text { stopping time } \\
\text { condition } \mathbf{T} \\
\text { Convergence is } \\
\text { always guaranteed }\end{array}$ & $\begin{array}{l}\text { The reached point is } \\
\text { not always an } \\
\text { equilibrium of the } \Sigma \text { - } \\
\text { interference game }\end{array}$ \\
$\begin{array}{l}\text { ID of } \\
\text { neighboring } \\
\text { nodes }\end{array}$ & $\begin{array}{l}\text { The reached point is } \\
\text { always an } \\
\text { equilibrium of the } \\
\text { M-interference game }\end{array}$ \\
\hline
\end{tabular}

(each player $i$ changes the starting strategy $P_{i}=0.5$ at most once) and it is distributed in the sense that it relies upon information that can easily be available at each node.

Table 2 reports a summary of the properties of the game heuristic (reported in Algorithm 1) and the DSP (reported in Algorithm 2) and shows the benefits of DSP in terms of convergence and stability of the reached point.

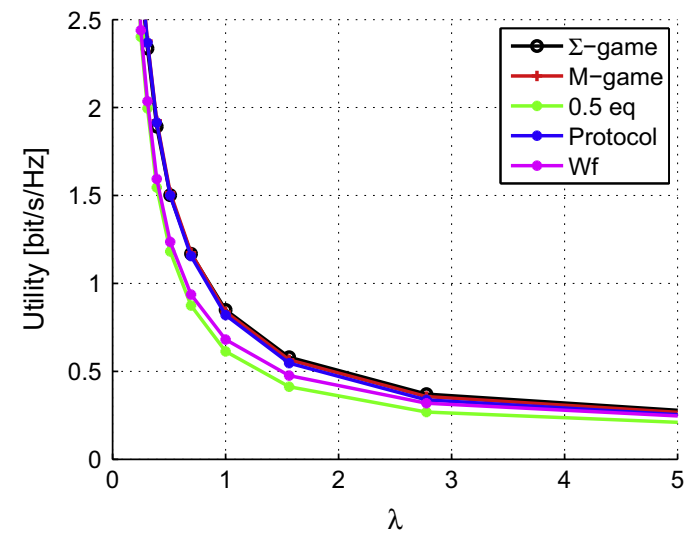

Fig. 18. Utility (Shannon rate) obtained with the different approaches varying $\lambda$.

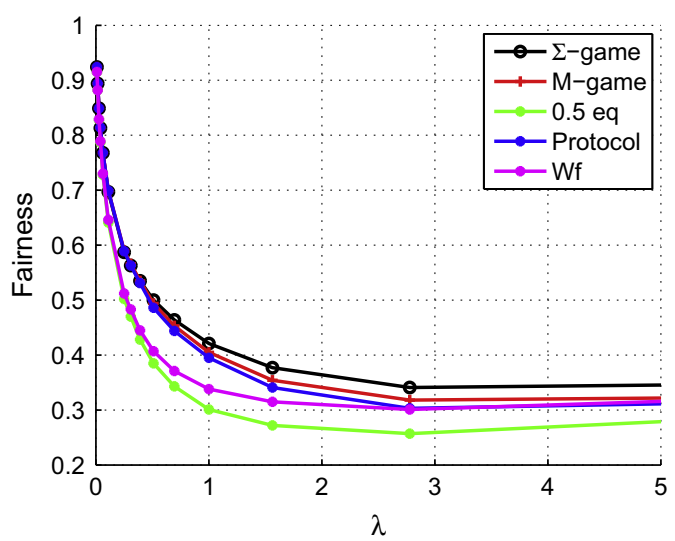

Fig. 19. Fairness obtained with the different approaches varying $\lambda$.

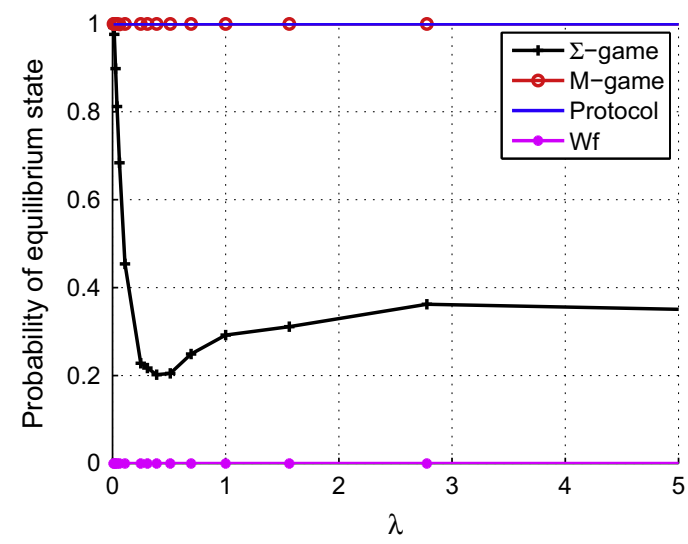

Fig. 20. Probability of reaching a Nash equilibrium at the termination.

The quality of the equilibria reached by the DSP is discussed hereafter through simulations. We evaluate the performance of the proposed protocol, considering the same scenario introduced before. We compare the different approaches in terms of utility, i.e., Shannon capacity, and fairness. Results are reported in Figs. 18 and 19. In 


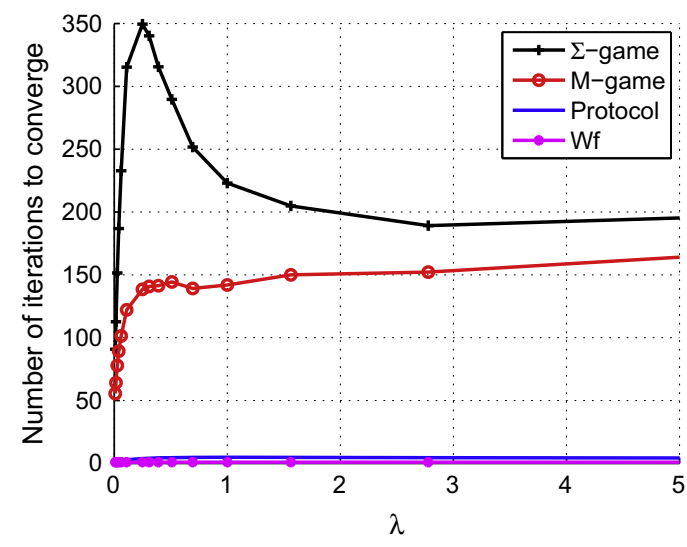

Fig. 21. Number of iterations to converge.

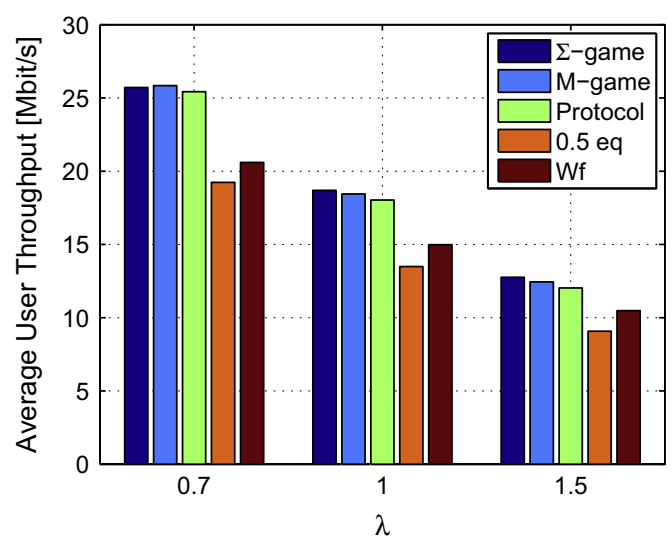

Fig. 22. Average throughput per user in WiFi scenario for different values of $\lambda$.

the same figures, we report the performance of: "0.5-equilibrium", in which each player plays the strategy 0.5. Also, we compare the results with a "water filling"-based approach (Wf). In this case, users start transmitting sequentially in a random order and adapt the power over the two bands treating as noise the users that already started their transmissions, without any further adaptation or coordination among users. Numerical results show that both the DSP and the GH improve the quality of the " 0.5 equilibrium", which is the reason why we introduce such mechanisms instead of letting all players simply play the strategy 0.5 .

Advantages of the proposed DSP are shown in Fig. 20 and Fig. 21, which show, respectively, the probability that the state reached at the termination is a stable point (by Nash equilibrium definition, according to the specific BR) and the number of iterations to converge. The $\Sigma$-interference game converges to a solution that satisfies the NE condition with probability that in general could be very far from one. In contrast, the M-interference game has a probability very close to one. Similarly, the DSP reaches a stable point with probability equal to one. In terms of converge time, the DSP dramatically reduces the number of iterations with respect to both the $\Sigma$-interference game and the $\mathrm{M}$-interference game. Note that the water filling policy converges fast but it never satisfies the equilibrium condition, having no coordination among the users.

Finally, we show the performance of the DSP as well as the other approaches in terms of network throughput. In particular, we consider the very same network topologies used so far for the analysis and defined in Section 6.1, we let each transmitter-receiver couple communicate according to the power policy derived by the different algorithms and we evaluate in such settings the per-user throughput assuming that the IEEE $802.11 \mathrm{~g}$ technology is adopted at the physical and MAC layers. Fig. 22 reports the average per-user throughput under different topologies (different values of the network spatial density parameter $\lambda$ ). The game heuristic and the DSP increase the network performance up to $25 \%$ with respect to both the water filling and the case in which the power is equally spitted over the two channels ("0.5-eq"). Moreover, the main benefit of the proposed DSP with respect to the game heuristics is in terms of convergence time. In average, the DSP solution is reached with less than 5 iterations, whereas the game heuristics may require some hundreds.

\section{Conclusion}

In this work, we analyze the spectrum sharing problem in ad hoc wireless networks where multiple pairs share the same portion of the spectrum. In this scenario, the "quality" perceived by each receiver is influenced by the behavior of the other transmitters, then we model the problem as a non-cooperative game.

First, we consider the scenario composed by 2 pairs of transmitter and receiver. We model this case as a 2-player game and we provide explicit characterization of the pure strategy Nash equilibria. Furthermore, due to the tight dependence of the equilibria with the position of the users and distances between interfering nodes, we analyze the problem using stochastic geometry, assuming that pairs are randomly dropped.

The 2-player game analysis becomes then fundamental for making the $N$-player game tractable. We consider both the $\Sigma$-interference game and the $\mathrm{M}$-interference game. Numerical evaluation shows that they are very close in terms of both strategy selection and user's utility, especially for the low $\lambda$ regime. Therefore, we concentrate on the $\mathrm{M}$-interference game, which can be analyzed using a directed-influence graph approach. In particular, we characterize the probability of being "coupled" and derive its value for $\lambda$ that goes to zero.

Finally, using the results of the theoretic models, we propose a distributed protocol for spectrum sharing in ad hoc networks. This allows to reach performance very close to the game, but with a significantly less number of iterations and probability of converging to an equilibrium point equal to one. 


\section{References}

[1] I. Malanchini, S. Weber, M. Cesana, Nash equilibria for spectrum sharing of two bands among two players, in: 2010 48th Annual Allerton Conference on Communication, Control, and Computing (Allerton), IEEE, 2010, pp. 783-790.

[2] I. Malanchini, S. Weber, M. Cesana, Stochastic characterization of the two band two player spectrum sharing game, in: 2012 10th International Symposium on Modeling and Optimization in Mobile, Ad Hoc and Wireless Networks (WiOpt), IEEE, 2012, pp. 343-348.

[3] P. Kyasanur, N.H. Vaidya, Capacity of multi-channel wireless networks: Impact of number of channels and interfaces, in: Proceedings of the 11th Annual International Conference on Mobile Computing and Networking, MobiCom '05, ACM, New York, NY, USA, 2005, pp. 43-57.

[4] G. Kulkarni, S. Adlakha, M. Srivastava, Subcarrier allocation and bit loading algorithms for OFDMA-based wireless networks, IEEE Trans. Mob. Comput. 4 (6) (2005) 652-662.

[5] R. Etkin, A. Parekh, D. Tse, Spectrum sharing for unlicensed bands, IEEE J. Sel. Areas Commun. 25 (3) (2007) 517-528.

[6] E. Altman, K. Avrachenkov, A. Garnaev, Transmission power control game with SINR as objective function, Network Control and Optimization, vol. 5425, Springer, Berlin, 2009, pp. 112-120.

[7] F. Wang, M. Krunz, S. Cui, Price-based spectrum management in cognitive radio networks, IEEE J. Sel. Top. Signal Process. 2 (1) (2008) 74-87.

[8] W. Yu, G. Ginis, J.M. Cioffi, Distributed multiuser power control for digital subscriber lines, IEEE J. Sel. Areas Commun. 20 (5) (2002) 1105-1115.

[9] R. Mochaourab, E. Jorswieck, Resource allocation in protected and shared bands: uniqueness and efficiency of Nash equilibria, in: Proc. GAMECOMM, Pisa, Italy, 2009, pp. 1-10.

[10] Z.-Q. Luo, J.-S. Pang, Analysis of iterative waterfilling algorithm for multiuser power control in digital subscriber lines, EURASIP J. Appl. Signal Process. 2006 (2005) 1-10.

[11] G. Scutari, D.P. Palomar, S. Barbarossa, The MIMO iterative waterfilling algorithm, IEEE Trans. Signal Process. 57 (5) (2009) 1917-1935.

[12] G. Scutari, D.P. Palomar, S. Barbarossa, Competitive design of multiuser MIMO systems based on game theory: a unified view, IEEE J. Sel. Areas Commun. 26 (7) (2008) 1089-1103.

[13] M. Baidas, A. MacKenzie, An auction mechanism for power allocation in multi-source multi-relay cooperative wireless networks, IEEE Trans. Wireless Commun. 11 (9) (2012) 3250-3260.

[14] R.D. Taranto, H. Yomo, P. Popovski, Two players non-cooperative iterative power control for spectrum sharing, in: Proc. IEEE PIMRC 2008, pp. $1-5$.

[15] J. Jia, Q. Zhang, A non-cooperative power control game for secondary spectrum sharing, in: Proc. IEEE ICC, 2007, pp. 5933-5938.

[16] Y. Wu, D.H.K. Tsang, Distributed power allocation algorithm for spectrum sharing cognitive radio networks with QoS guarantee, in: Proc. IEEE INFOCOM, 2009, pp. 981-989.

[17] C. Tekin, M. Liu, R. Southwell, J. Huang, S. Ahmad, Atomic congestion games on graphs and their applications in networking, IEEE/ACM Trans. Netw. 20 (5) (2012) 1541-1552.

[18] X. Chen, J. Huang, Spatial spectrum access game: Nash equilibria and distributed learning, in: Proceedings of the Thirteenth ACM International Symposium on Mobile Ad Hoc Networking and Computing, MobiHoc '12, ACM, New York, NY, USA, 2012, pp. 205-214.

[19] E.V. Belmega, B. Djeumou, S. Lasaulce, Power allocation games in interference relay channels: existence analysis of Nash equilibria, EURASIP J. Wirel. Commun. Netw. 2010 (2010) 114:1-114:20.

[20] P. Mertikopoulos, E.V. Belmega, A.L. Moustakas, S. Lasaulce, Distributed learning policies for power allocation in multiple access channels, IEEE J. Sel. Areas Commun. 30 (1) (2012) 96-106.

[21] L.H. Grokop, D.N.C. Tse, Spectrum sharing between wireless networks, IEEE/ACM Trans. Netw. 18 (5) (2010) 1401-1412.

[22] G. Hosseinabadi, M.H. Manshaei, J.-P. Hubaux, Spectrum sharing games of infrastructure-based cognitive radio networks, Technical Report LCA-REPORT-2008-027.

[23] M. Bennis, M.L. Treust, S. Lasaulce, M. Debbah, J. Lilleberg, Spectrum sharing games on the interference channel, in: Proc. GameNets, Istanbul, Turkey, 2009, pp. 515-522.

[24] M. Haenggi, J. Andrews, F. Baccelli, O. Dousse, M. Franceschetti, Stochastic geometry and random graphs for the analysis and design of wireless networks, IEEE J. Sel. Areas Commun. 27 (7) (2009) 10291046. stochastic SI.

[25] F. Fu, M. Van der Schaar, Learning to compete for resources in wireless stochastic games, IEEE Trans. Veh. Technol. 58 (4) (2009) 1904-1919. stochastic SI.

[26] S. Weber, J.G. Andrews, Transmission capacity of wireless networks, Found. Trends ${ }^{\circledR}$ Netw. 5 (2-3) (2012) 109-281.

[27] F. Baccelli, P. Bermolen, Extremal versus additive matern point processes, Queueing Syst. 71 (1-2) (2012) 179-197.

[28] I. Malanchini, Game theoretic models for resource sharing in wireless networks (Ph.D. thesis), Drexel University, Philadelphia, 2012.

[29] E. Anshelevich, A. Dasgupta, J. Kleinberg, E. Tardos, T. Wexler, T. Roughgarden, The price of stability for network design with fair cost allocation, in: IEEE FOCS, 2004, pp. 59-73.

[30] E. Koutsoupias, C. Papadimitriou, Worst-case equilibria, in: 16th Annual Symposium Theoretical Aspects Computer Science. STACS, Springer, Trier, Germany, 1999, pp. 404-413.

[31] R. Jain, D. Chiu, W. Hawe, A quantitative measure of fairness and discrimination for resource allocation in shared computer systems, Tech. Rep. TR-301, DEC Research Report, September 1984.

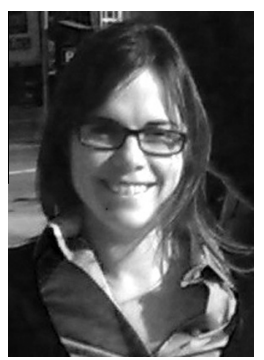

Ilaria Malanchini received the B.S. and M.S. degrees in telecommunications engineering from Politecnico di Milano, Italy, in 2005 and 2007, respectively. In December 2011, she received the Ph.D. degree in electrical engineering from both Drexel University (Philadelphia) and Politecnico di Milano. She joined Alcatel-Lucent Bell Labs in 2012. Her research interests focus on optimization models, mathematical programming, game theory, and stochastic geometry, with the application of these techniques to wireless network problems such as network selection and spectrum sharing.

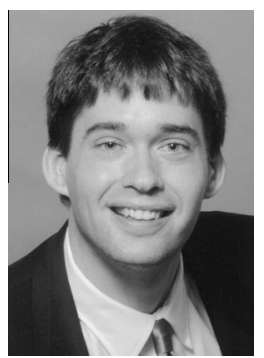

Steven Weber received his B.S. degree in 1996 from Marquette University in Milwaukee, WI, and his M.S. and Ph.D. degrees from The University of Texas at Austin in 1999 and 2003 respectively. He joined the Department of Electrical and Computer Engineering at Drexel University in 2003 where he is currently an associate professor. His research interests are centered around mathematical modeling of computer and communication networks, specifically streaming multimedia and ad hoc networks.

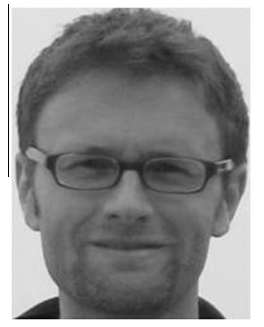

Matteo Cesana is currently an Assistant Professor with the Dipartimento di Elettronica, Informazione e Bioingegneria of the Politecnico di Milano, Italy. He received his M.S. degree in Telecommunications Engineering and his Ph.D. degree in Information Engineering from Politecnico di Milano in July 2000 and in September 2004, respectively. From September 2002 to March 2003 he was a visiting researcher at the Computer Science Department of the University of California in Los Angeles (UCLA). His research activities are in the field of design, optimization and performance evaluation of wireless networks with a specific focus on wireless sensor networks and cognitive radio networks. Dr. Cesana is an Associate Editor of the Ad Hoc Networks Journal (Elsevier). 